\title{
Using Active Ultrasonics to Measure Wheel-Rail Contact During a Running-in Period
}

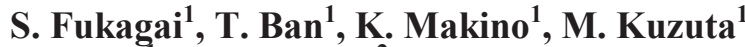 \\ H. Chen ${ }^{1}$, H. Brunskill ${ }^{2}$ and R.S. Dwyer-Joyce ${ }^{2}$ \\ ${ }^{1}$ Railway Technical Research Institute, Tokyo, Japan \\ ${ }^{2}$ Leonardo Centre for Tribology, University of Sheffield, Sheffield \\ United Kingdom
}

\begin{abstract}
Flange climb derailment is most likely to occur during the wheel-rail running-in phase, such as just after the railway vehicle wheels have been re-profiled or during morning rush hour. It is thought that the underlying cause is an increase in traction due to changes in the contact between wheel and rail. However, the mechanism of this increase in traction remains a subject of continuing controversy. Active ultrasonic measurements have been effectively used to measure wheel-rail contact conditions, particularly the contact stiffness. In this study, the ultrasonic method is used to characterise the wheel-rail contact during running-in whilst the surface roughness of the wheel reduces due to cyclic loading against the rail. An array of sensors was mounted behind the wheel flange so as to reflect ultrasound directly from the contact. An initial investigation was carried out to understand the measurement resolution of this ultrasonic array transducer. A static measurement was then performed by traversing the ultrasonic array transducer across the wheel when statically loaded against the rail. Following this, the same array transducer was fixed to the wheel and dynamic measurements were carried out. These measurements were cyclic to investigate the effect on changing surface topography. From this, the relationship between the slip and the contact stiffness was investigated. It was found that an increase of contact stiffness was measurement after slip motion was recognized. Both the static and dynamic measurements showed a difference in the wheel-rail contact conditions. In the case of the rolling condition, the peak position of the contact stiffness in the contact area shifted from the centre of the contact to the exit side. From the cyclic rolling tests, it was observed that the change in surface topography resulted in an increase in interfacial contact stiffness. This phenomenon indicates that there is an increase in real area of contact between the wheel flange and the rail gauge corner during running-in.
\end{abstract}

Keywords: flange climb derailment, running-in, contact stiffness, real contact area, ultrasonic measurements. 


\section{Introduction}

When a railway vehicle negotiates a sharp curve or a turnout on railway tracks, the wheel flange contacts the rail gauge corner to guide the running direction. The contact at this location is severe with high stress, slip and friction. In some cases, it can lead to flange climb derailments. Several derailments have occurred relatively soon after the re-profiling of the wheel [1-3] or during morning rush hour [4]. Some experimental results using a twin-disk test machine showed that the coefficient of friction reached its peak during running-in $[5,6]$. As the wheel flange and rail gauge corner experience highly loaded cyclic rolling, the surface topography changes $[7,8]$ and therefore the real area of contact and contact stiffness alters dramatically during running-in. Therefore, it is important to understand how these contact conditions evolve over time to understand the potential mechanisms for flange climb derailment. However, the difficulty in obtaining accurate non-destructive interfacial measurements has hindered systematic experimental investigations. An increased understanding of these effects might inform rail service providers about optimal wheel profiling methods and lubrication programs to reduce the likelihood of flange climb derailment.

Pressure-sensitive films can be used to observe contact area and stress. However, these films may cause over-prediction of the contact area owing to thickness of the film and changes in tangential force due to its different frictional properties. Practical implementation is also difficult because the film disintegrates under the high-pressure and shear between the wheel and the rail [9]. Recently, ultrasonic techniques have been used to observe the contact between wheel and rail [10-20]. This technique can be used to non-invasively and directly observe the contact. However, there are spatial resolution limits, considerations of transducer positioning to ensure the soundwaves reflect off the area of interest. When an ultrasonic wave strikes the interface between the wheel and rail, it is partially transmitted and partially reflected. The proportion of the wave reflected depends on the stiffness of the contact. Determining real area of contact and friction from the contact stiffness is not uniquely possible, but empirical correlations have shown that for most practical loads, there is a linear relationship between contact stiffness and contact pressure $[15,16,18]$. This approach has been used to determine the contact pressure distribution in wheel-rail contacts, and the influence of wear, roughness and surface defects on the contact patch $[12,14,16]$.

Recently, the dynamic measurement of a rolling contact condition has been achieved [20]. These results were based on one- or two-time contact tests and changes caused by cyclic rolling contact were not investigated. Information that considers the change in surface condition and contact under cyclic loading, is one of the key factors needed to investigate the tribological mechanism of flange climb derailment.

In this study, ultrasonic reflection was used to evaluate changes in the contact condition, particularly the contact stiffness over time as running-in occurs. The contact stiffness is a function of the surface topography, the surface material properties and the applied pressure. The experiments were performed using a fullscale wheel/rail contact-testing machine. A focused ultrasonic array probe consisting 
of sixty-four ultrasonic elements was mounted in a water bath behind the wheel flange. An initial body of work was carried out to characterise the transducer resolution. Static measurements were achieved by scanning the transducer across the contact. Following this, the transducer was mounted on the wheel and dynamic tests were performed building up a two-dimensional contact profile as the array passed over the contact. The aim was to understand the influences of slip, and cyclic rolling contact on the gauge corner contact.

This paper follows from previous work of Fukagai et al. [21], but extends the approach to include the analysis of contact stiffness variation; and the influence of the relative slip between wheel and rail. Slip is an important factor because it leads to increased plastic flow and dramatic changes in surface roughness. The static contact condition was evaluated with the gradual increase of lateral tangential force under the constant normal force to investigate the relationship between the slip motion and contact stiffness.

\section{Principle of measurement}

When an ultrasonic pressure wave strikes an interface between the two materials, a part of the incident ultrasonic wave is transmitted through the interface and the other portion is reflected from the interface. For an atomically perfect interface of two dissimilar materials, the proportion of the reflected wave, described by the reflection coefficient $R$, is dependant on the acoustic impedance mismatch between the two materials and is given in [22]

$$
R=\frac{z_{2}-z_{1}}{z_{2}+z_{1}}
$$

where $z_{1}$ and $z_{2}$ are the acoustic impedances (which are the products of density and acoustic velocity) of the contacting materials. Therefore, for two perfectly bonded identical materials, the interface would have no reflection $\left(z_{1}=z_{2}, R=0\right)$ and the entire wave is transmitted (without any losses). Conversely, an ultrasonic wave is almost completely reflected at an interface between two materials with substantially different acoustic impedances, as in the case of a solid and a gas $(R \approx 1)$.

Real engineering interfaces are inherently rough and micro and macroscopic air gaps are formed at an interface. Presuming the length of the ultrasonic wave is long relative to the size of the air gaps the whole interface behaves as a single reflector and therefore $R$ is also dependant on the contact stiffness of the interface.

The contact stiffness, $K$, is a function of the surface topography, surface material properties and the contact pressure. As the surface topography changes due to elastic and plastic deformation, the measured reflection coefficient $R$ will change accordingly as shown in Figure 1. In principle, the degree of conformity at the interface can be determined by measuring the reflection coefficient of the ultrasonic wave. 


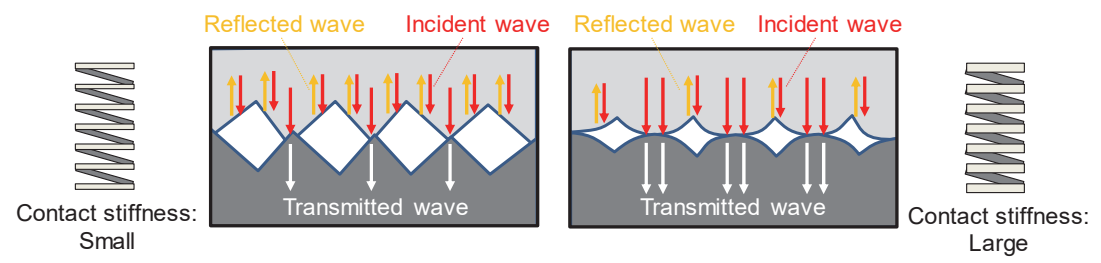

Figure 1: Principle of measurement

Schoenberg [22] used an interface 'spring model' showed that the reflection coefficient is then obtained from

$$
R=\frac{z_{2}-z_{1}+i \omega\left(z_{1} z_{2} / K\right)}{z_{2}+z_{1}+i \omega\left(z_{1} z_{2} / K\right)},
$$

where $\omega$ is the angular frequency of the ultrasonic wave and $K$ is the interfacial contact stiffness. In the case of present work, the materials on both sides of the interface are similar $\left(z_{1}=z_{2}=z\right)$, then Equation (2) reduces to:

$$
|R|=\frac{1}{\sqrt{1+(2 K / \omega z)^{2}}} .
$$

Therefore, if the reflection coefficient, $R$, can be experimentally obtained, the contact stiffness, $K$ can be estimated. Although some research $[15,16,18]$ has been carried out to seek the contact pressure using a linear relationship between the contact stiffness and the contact pressure, it is only applicable under the assumption of a constant roughness and material properties. In this paper, the effect due to the change in surface roughness and material properties with cyclic contact was investigated so it was not possible to calculate the contact pressure.

Many researchers [10-20] have obtained the reflection coefficient $R$ as the ratio of the reflected ultrasonic wave amplitude under load, $H$, to that when unloaded, $H_{0}$ (the reference).

$$
R=\frac{H}{H_{0}} .
$$

When unloaded the contact is effectively steel against air and so the wave is fully reflected and $H_{0}$ is equivalent to the incident wave amplitude. This is a simple practical way to obtain the reflection coefficient and removes the influence of transducer characteristics and ultrasonic wave scattering. 


\section{Experiments}

\subsection{Ultrasonic measurement system}

An ultrasonic measuring instrument consisting of an array transducer (Olympus, 10L64-I1) and pulsing-receiving-digitising instrumentation (Olympus, OmniScan MX) was employed for this work. The transducer was the immersion type and the frequency was $10 \mathrm{MHz}$. The transducer consisted of sixty-four linear elements spaced at regular intervals of $0.5 \mathrm{~mm}$. These elements synthesize multiple beam components and emit multiple ultrasonic waves to form a single wave traveling in the intended direction. And the receiving function combines inputs from multiple elements and performs a single display in the same way. Controlling the excitation timing of these elements enables beam focusing to an arbitrary depth, the beam steering in the arbitrary direction and the high-speed data acquisition in the array direction of the elements. A curved wedge was used to adjust the focus on the short side of assembly, where no array existed.

The signals of the reflected wave were rectified to all be a positive value and the peak value of these rectified signals within the selected depth range were acquired.

\subsection{Experimental devices}

Figure 2 shows a schematic illustration of the calibration test setup used to assess the transducer resolution. The set-up consisted of the ultrasonic array transducer mounted on a linear scanning system. A steel block section of $15 \mathrm{~mm}$ thickness was cut from a full wheel and was mounted on another cuboid steel block. The array transducer was focused onto the contacting interface. The path length in water to achieve a focus on the back face of the steel block was $12 \mathrm{~mm}$. These values are the same as with the full-scale measurement. The transducer records a linear scan of reflection coefficient. As it is traversed across the contact, the result is a two-dimensional scan of the interface. The actual nominal contact area is known from the cuboid block geometry, allowing a comparison between the measured area thus informing the resolution of the system.

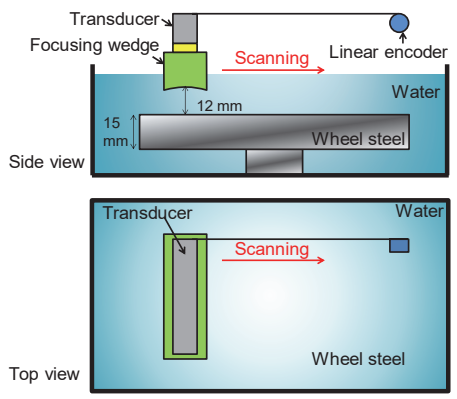

Figure 2: Schematic illustration of the calibration steel block for the detection of the spread of ultrasonic wave 
Figure 3 shows a schematic illustration of the full-scale testing machine, which is equipped with an actual wheel and rail loaded upside down on it. It can apply a vertical load of up to $50 \mathrm{kN}$, a lateral load of up to $40 \mathrm{kN}$ and a rail longitudinal load of up to $20 \mathrm{kN}$ using hydraulic cylinders to push the rail. The rail is moved at a velocity of up to $100 \mathrm{~mm} / \mathrm{s}$ in the longitudinal direction. Although the wheel exhibits no driving performance, it is rotated by the force generated due to the friction between the wheel and the rail. The angle of attack is changeable from -3-degree to +3 -degree by adjusting the plate on which the rail is mounted. A water bath was mounted on a modified wheel. The immersion transducer was mounted on a scanning system for the static measurements and was fixed in place for the dynamic measurements.

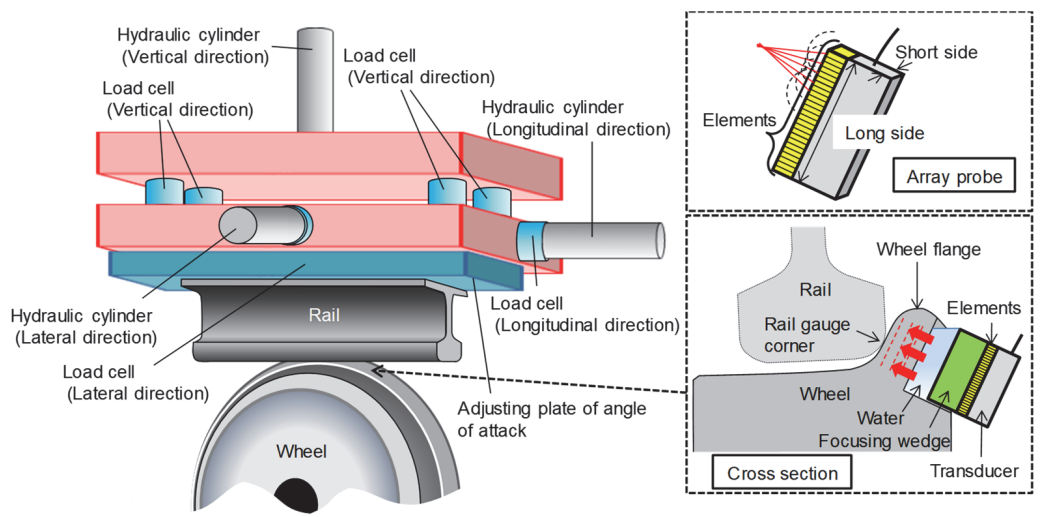

Figure 3: Schematic of the full-scale testing machine

Figure 4 shows the wheel flange forces acting on the rail gauge corner and the transducer position. Figure 5 shows the profiles of the wheel and the rail that were used in the tests. Both the wheel and the rail were new unused and the profile of the wheel was "modified arc tread" and that of the rail was " $50 \mathrm{kgN}$ ". The rail contacts with the wheel flange at one contact patch around the straight section of the wheel flange. The rail was mounted on the testing machine without an angle of cant. In the test machine a vertical load $P$ and lateral load $Q$ were applied; but for defining the test conditions these were used to determine the normal force $N$ and the lateral tangential force $F_{y}$ with the following equations:

$$
\begin{aligned}
& N=P \cos \alpha+Q \sin \alpha, \\
& F_{y}=P \sin \alpha-Q \cos \alpha,
\end{aligned}
$$

where $\alpha$ is the contact angle ( $65^{\circ}$ in this experiment).

The longitudinal tangential force $F_{x}$ was set independently of the vertical load $P$ and lateral load $Q$. It should be noted that the tangential force coefficient, which is described later, means $F_{y} / N$. As shown in Figure 4, the ultrasonic wave was pulsed 
from a transducer mounted on the wheel flange. The backside of the wheel flange was cut to maintain a constant water path distance between the ultrasonic transducer and the straight section of the flange surface. As in the calibration specimen described above, the wheel was $15 \mathrm{~mm}$ thick and the transducer path length in water was 12 $\mathrm{mm}$.

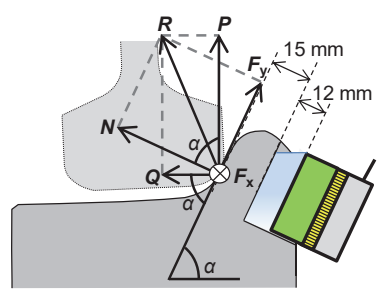

Figure 4: Illustration of the wheel flange forces acting on the rail gauge corner and transducer position
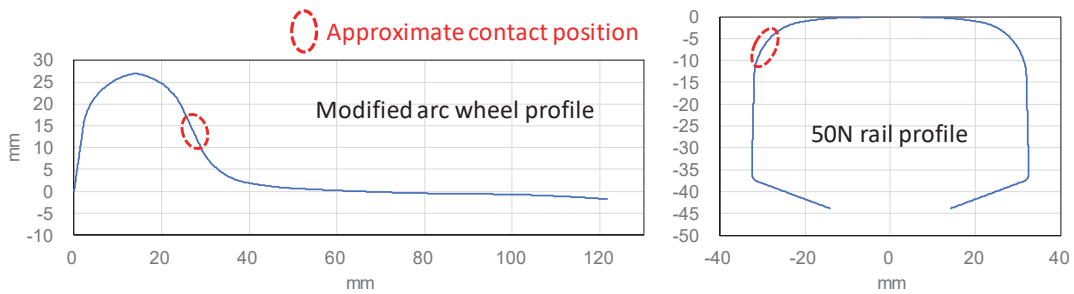

Figure 5: Profiles of the wheel and the rail showing the approximate location of the contact region

\subsection{Static measurement method}

Figure 6 shows schematics and a photograph of the static measurement method. The transducer was attached to an electric linear stage set behind the wheel flange. The rail was pushed to the wheel flange by predefined lateral and vertical loads. The static contact condition between the wheel flange and the rail gauge corner was scanned when the transducer passed through the contact patch via the linear motion of the electric stage. The linear encoder attached to the transducer holder measured its position. In this way the distribution of $R$ on the rectangular coordinate was obtained from an electronically scanned position by the array along the lateral direction of the wheel flange ( $y$-axis, $F_{y}$ direction in Figure 4$)$ and the transducer movement distance along the longitudinal direction of the rail ( $x$-axis). The sampling interval in the lateral and longitudinal directions was 0.25 and $0.2 \mathrm{~mm}$, respectively. Although the spacing of the elements in the array was $0.5 \mathrm{~mm}$, the sampling interval was improved to 0.25 $\mathrm{mm}$ different combinations of group elements. Figure 7 shows an example of this. In this case, a sample interval of $0.25 \mathrm{~mm}$ is obtained by using 7 elements and 8 elements alternately. 
Initially, the set-up condition of the load for comparison with the rolling measurement was determined as follows: The normal force was 15 or $25 \mathrm{kN}$ and the lateral tangential force was $0 \mathrm{kN}$ (No. 1-1, 1-2). Then the lateral tangential force was increased stepwise with a fixed normal force, either $15 \mathrm{kN}$ or $25 \mathrm{kN}$, to investigate the influence of slip (No. 2-1, 2-2). The angle of attack was set at $0^{\circ}$ for the entire test.
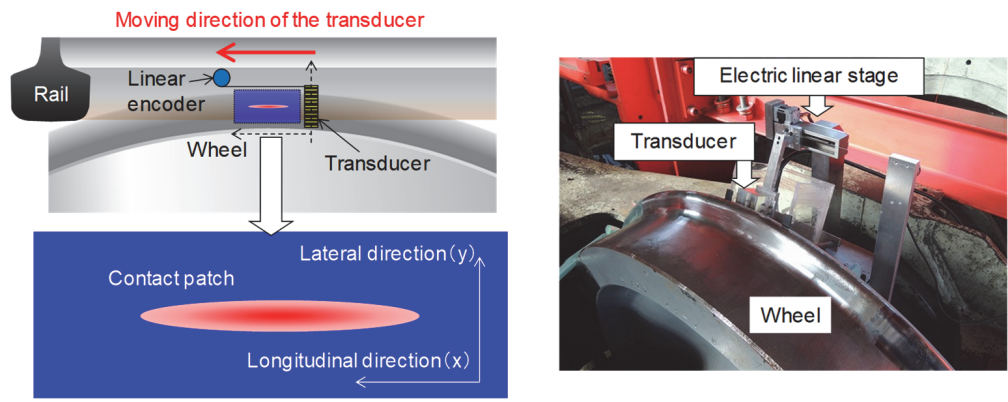

Figure 6: Schematics and photograph of the method for static measurement

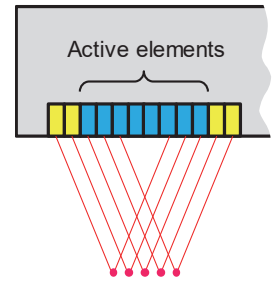

Without combination

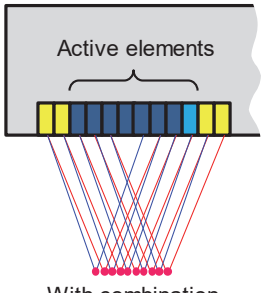

With combination

Figure 7: An example of the combination of the different grouped elements to increase sampling interval

\subsection{Rolling measurement method}

Figure 8 shows schematics and a photograph for the rolling measurement method. Again, the transducer was set directly behind the wheel flange. The rail was pushed longitudinally after it contacted the wheel flange and bidirectionally moved along with the longitudinal direction. The frictional force between the wheel and rail caused the wheel to rotate. The rolling contact between the wheel flange and the rail gauge corner was scanned when the transducer passed through the contact patch as the wheel rotated. The rotary encoder attached to the wheel tread that rotated with the wheel measured the rotational angle of the wheel. In this way, the distribution of $R$ on the polar coordinate was obtained from the transducer scanning position along the radial direction ( $y^{\prime}$-axis) and rotational distance (angle) ( $x^{\prime}$-axis) of the wheel. An array of $R$ data could be obtained at every constant pitch of rotation angle that was detected by the rotary encoder. By coordinate change using the information of the position on the radial direction where the transducer was set and the rotation angle, the distribution of $R$ in rectangular coordinates could be obtained. The sample interval of $R$ and the 
rotational angle interval were $0.25 \mathrm{~mm}$ and $0.011^{\circ}$, respectively. After the coordinate change, the sample interval in the lateral direction (y-axis) and longitudinal direction ( $x$-axis) were 0.25 and $0.2 \mathrm{~mm}$, respectively.

The load set-up for the rolling measurement was determined as follows: normal forces of 15 and $25 \mathrm{kN}$ and a lateral tangential force of $0 \mathrm{kN}$. The rolling measurement was carried out not only during one-time rolling (No. 3-1, 3-2) but also during cyclic rolling (No. 4-1, 4-2) to investigate the changes of contact condition during runningin. The number of cyclic rolls was 10 or 19 (5 or 9.5 round trips). The wheel flange surface and rail gauge corner were smoothed using sandpaper before each test. The angle of attack was set at $0^{\circ}$.

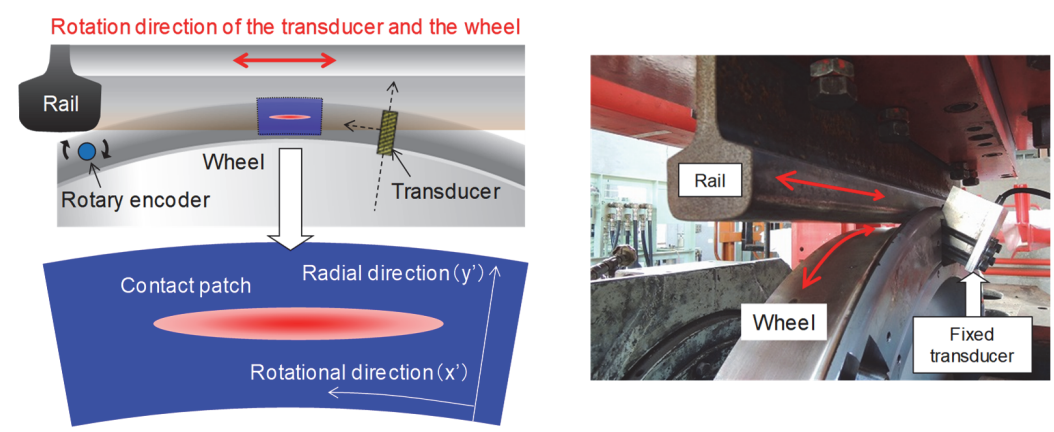

Figure 8. Schematics and photograph of the method for dynamic measurement.

\section{Experimental results}

\subsection{Measurement of spatial resolution}

The distribution of the reflection coefficient $R$ on the bottom of steel block that had a cuboid projection (Figure 2) was measured and the spread of the ultrasonic wave was investigated. Figure 9 shows the distribution of $R$. Here, the value of $R$ that is larger than a threshold value, $R_{t}$, was regarded as the background and the area was filled. $R_{t}$ is defined by the following equation:

$$
R_{t}=R_{a}-3 s
$$

where $R_{a}$ is the average value in the area, which is not within the square projection, and $s$ is the standard deviation in the same area. As shown in Figure 9, the region of contact can clearly be seen. However, the relatively large size of each transducer element means that the sharps edges are effectively blurred; this limits spatial resolution. The cross sections through the $R$ data show the spread of the ultrasonic wave was approximately $2-3 \mathrm{~mm}$ either side of the $x$ - and $y$-axes. This indicates the anticipated spatial resolution of this method. 

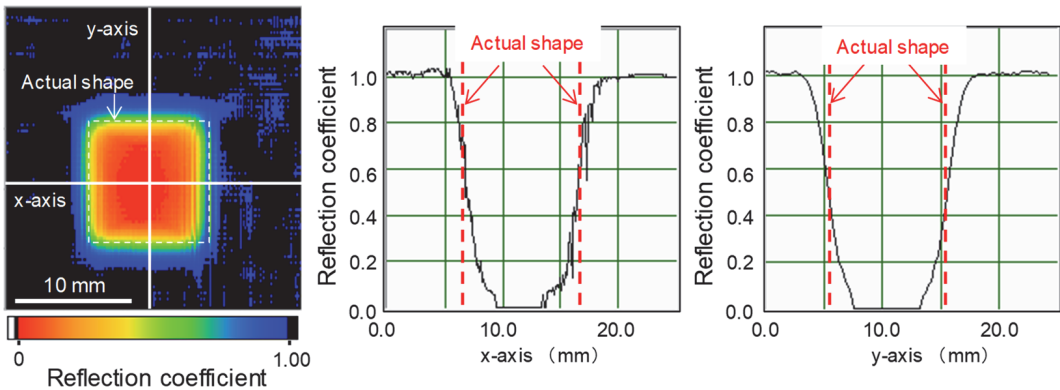

Figure 9: Distribution of $R$ measured from the calibration test block shown in Figure 2 .

\subsection{Static contact results}

Table 1 shows the static measurement test parameters. The test series No.1 and the series No. 2 were conducted contiguously without separating the wheel and rail. The wheel flange surface and rail gauge corner were smoothed using sandpaper (\#80) to initialize the surface condition; and the roughness was measured before each test. Series No.1 has static contact under only applied normal force; whilst series No. 2 is static contact with applied lateral tangential force.

\begin{tabular}{|c|c|c|c|c|c|}
\hline \multicolumn{2}{|c|}{ Test Name } & No. 1-1 & No. 1-2 & No. 2-1 & No. 2-2 \\
\hline \multicolumn{2}{|c|}{ Normal force, $N[\mathrm{kN}]$} & 15.1 & 24.9 & $14.9 \sim 15.1$ & $24.8 \sim 25.3$ \\
\hline \multicolumn{2}{|c|}{ Lateral tangential force $F_{y}[\mathrm{kN}]$} & -0.1 & -0.1 & $0.0 \sim 7.9$ & $0.0 \sim 12.6$ \\
\hline \multicolumn{2}{|c|}{ Longitudinal tangential force, $F_{x}[\mathrm{kN}]$} & -0.1 & -0.4 & $-0.3 \sim 0.0$ & $-0.3 \sim 0.1$ \\
\hline \multicolumn{2}{|l|}{$F_{y} / N$} & -0.01 & 0.00 & $0.00 \sim 0.52$ & $0.00 \sim 0.51$ \\
\hline \multirow{2}{*}{$\begin{array}{c}\text { Surface roughness } \\
\text { before test }[\mu \mathrm{m}]\end{array}$} & wheel & \multicolumn{2}{|c|}{0.52} & \multicolumn{2}{|c|}{0.38} \\
\hline & rail & \multicolumn{2}{|c|}{0.89} & \multicolumn{2}{|c|}{1.2} \\
\hline
\end{tabular}

Table 1: Test parameters for the static measurement

Figure 10 shows the distribution of the contact stiffness that was measured under the static contact condition with the normal forces of $15 \mathrm{kN}$ (No. 1-1) and $25 \mathrm{kN}$ (No. 1-2). The theoretical contact pressure distribution is also plotted in Figure 10. For the theoretical results, the contact area was calculated according to the 'exact theory' established by Kalker [23]. For the calculation, the smooth design profiles were used and the size of grid was $0.5 \mathrm{~mm}$ (x-axis) and $0.02 \mathrm{~mm}$ (y-axis) in the case of $15 \mathrm{kN}$ and $0.5 \mathrm{~mm}$ (x-axis) and $0.023 \mathrm{~mm}$ (y-axis) in the case of $25 \mathrm{kN}$. Here, the background area (defined in Section 4.1) has been shaded to compare the contact stiffness maps with the calculated contact pressure distribution. The measured contact stiffness has a greater area compared with the theoretical results. It can be seen that the contact region is blurred by out by approximately $2-3 \mathrm{~mm}$ in each direction. This agrees well with the spread of the ultrasonic wave determined from the calibration experiment of 
section 4.1. The value of contact stiffness increased as the measured point approached the ellipse centre; the distribution of contact stiffness correlated well with that of the contact pressure. This agrees with previous studies that suggest a direct or indirect relationship between $R$ and the contact pressure [10,14, 15, 16, 24].
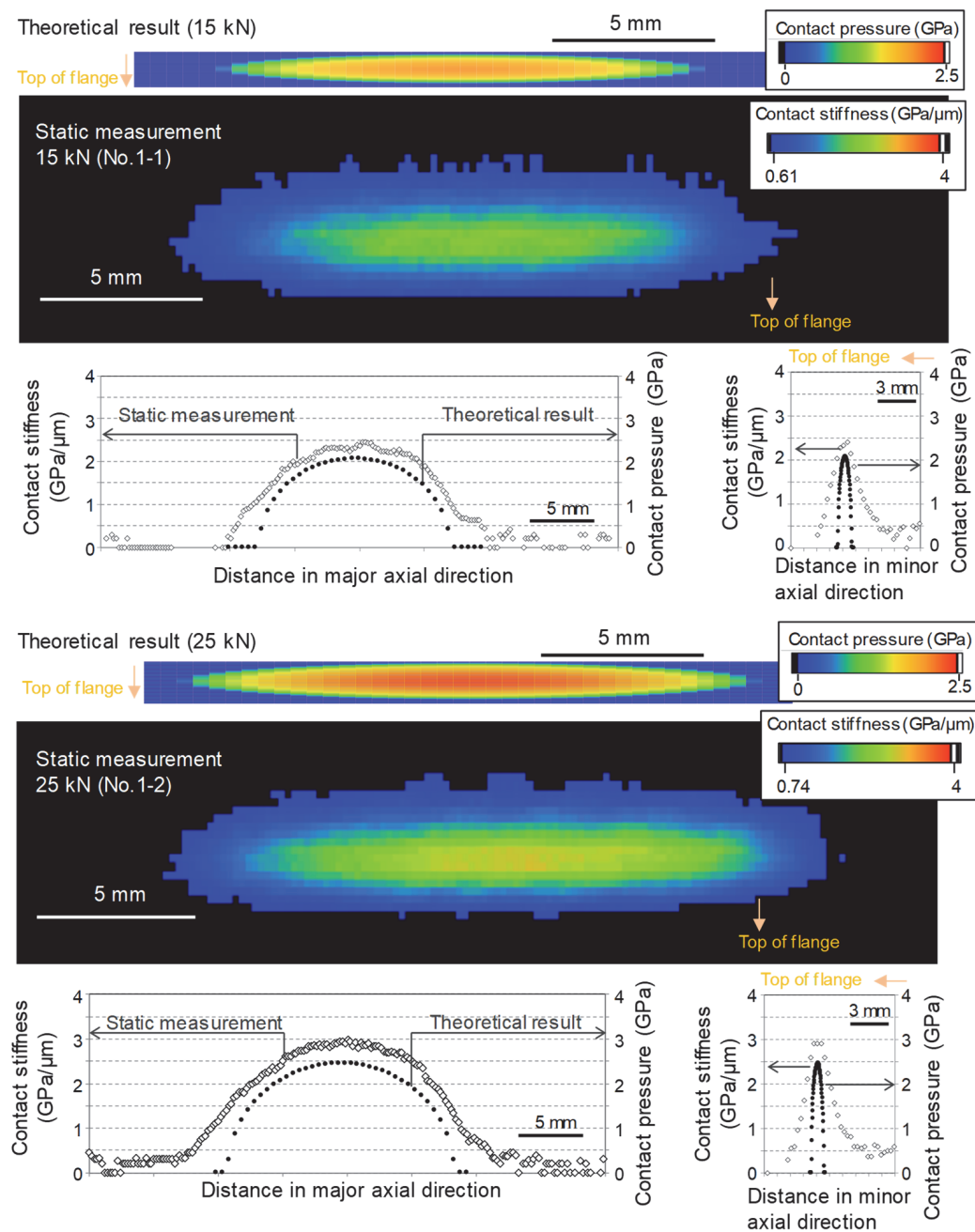

Figure 10: Distribution of measured contact stiffness under static contact with normal forces of 15 (No. 1-1: upper) and $25 \mathrm{kN}$ (No. 1-2: lower) and the theoretical distribution of contact pressure for comparison

Figure 11 shows the distribution of contact stiffness which was measured under the static contact condition of the normal force equalling $15 \mathrm{kN}$ or $25 \mathrm{kN}$ with the addition of lateral tangential force from 0.0 to 0.5 in the tangential force coefficient $F_{y} / N$ (No. 
2-1, 2-2). Here, tangential force coefficient $F_{y} / N$ is defined as the ratio of the tangential force to the normal force. The peak value increases with the tangential force coefficient $F_{y} / N$.

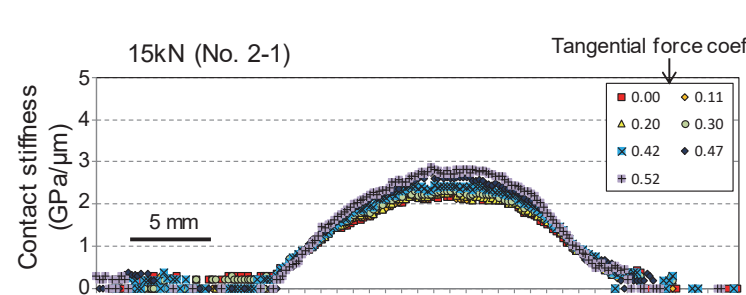

Distance in major axial direction

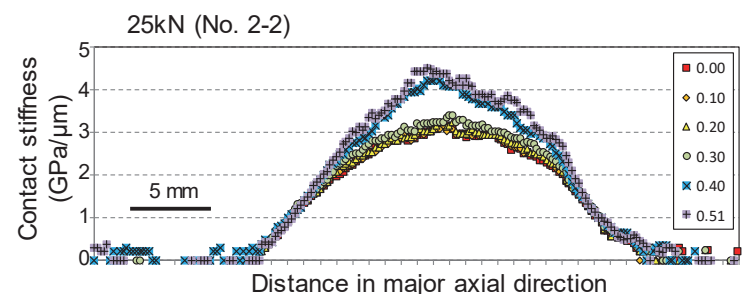

Distance in major axial direction
Direction of tangential force $\longrightarrow$

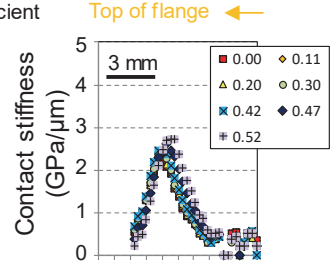

Distance in minor axial direction

Direction of tangential force $\longrightarrow$

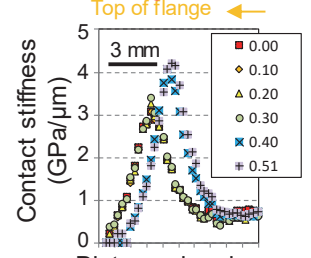

Distance in minor axial direction

Figure 11: Plots of measured contact stiffness under static contact with normal force of $15 \mathrm{kN}$ (No. 2-1: upper) and $25 \mathrm{kN}$ (No. 2-2: lower) and the addition of lateral tangential force from 0.0 to 0.5 in the tangential force coefficient

The plots show that interface stiffness increases with applied lateral force. For elastic smooth bodies of identical materials in contact (following Hertz assumptions) the normal and shear tractions are independent; so increasing the lateral force would not affect the contact pressure (and hence stiffness). Clearly some non-Hertzian effect is taking place here.

Figure 12 shows the relationship between the tangential force coefficient and the contact stiffness. Here, the mean contact stiffness is the mean value over the nominal contact area. The sum of the contact stiffness is the sum over the nominal contact area which has been normalized by the sum in the case where $F_{y} / N=0$. The nominal contact area has been defined as the cells where $R$ is smaller than the background value, $R_{\mathrm{t}}$ which was defined in Section 4.1. The plot shows that the change in mean contact stiffness is small up to $F_{y} / N=0.3$; and then significantly increases over $F_{y} / N=0.4$. Figure 11 showed that there were slight slips along with the minor axial direction over 0.4 in $F_{y} / N$. The results indicate that increased lateral stress causes asperity plastic flow to occur and greater conformity between the wheel and rail and hence the contact stiffness to increase. 


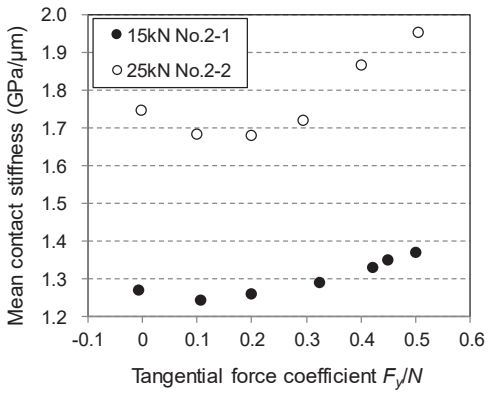

(a) Mean contact stiffness

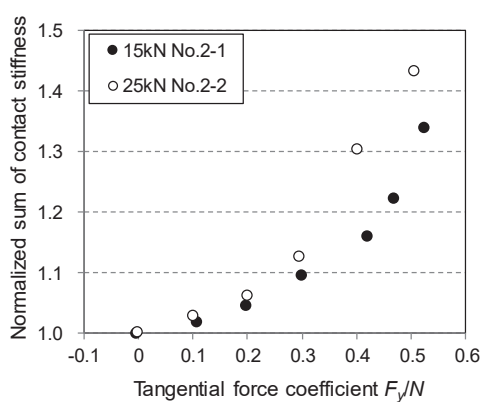

(b) Normalized sum of contact stiffness

Figure 12: The relationship between (a) mean contact stiffness (b) summed contact stiffness, and the tangential force coefficient

\subsection{Rolling contact results}

Table 2 shows the rolling measurement test parameters. The wheel flange surface and rail gauge corner were smoothed using sandpaper and the roughness was measured before each test. Here, the grade of the sandpaper in the Test No. 3 was selected \#80 to be same with Test No.1 and No.2. On the other side, that in Test No. 4 was selected \#30 to focus on the change with the cyclic contacts and to obtain rougher initial surface.

\begin{tabular}{|c|c|c|c|c|c|}
\hline \multicolumn{2}{|l|}{ Test name } & No. 3-1 & No. 3-2 & No. 4-1 & No. 4-2 \\
\hline \multicolumn{2}{|c|}{ Normal force, $N[\mathrm{kN}]$} & 15.3 & 24.4 & $15.1 \sim 15.7$ & $24.6 \sim 25.4$ \\
\hline \multicolumn{2}{|c|}{ Lateral tangential force, $F_{y}[\mathrm{kN}]$} & 0.5 & 0.2 & $0.1 \sim 1.8$ & $0.2 \sim 1.0$ \\
\hline \multicolumn{2}{|c|}{ Longitudinal tangential force, $F_{x}[\mathrm{kN}]$} & 0.5 & 0.6 & $-0.1 \sim 0.5$ & $-0.2 \sim 0.6$ \\
\hline \multicolumn{2}{|c|}{$F y / N$} & 0.03 & 0.01 & $0.01-0.11$ & $0.01-0.04$ \\
\hline \multirow{2}{*}{$\begin{array}{l}\text { Surface roughness }(\mathrm{Ra}) \\
\text { before test }[\mu \mathrm{m}]\end{array}$} & wheel & 0.57 & 0.58 & 0.93 & 0.86 \\
\hline & rail & 0.78 & 1.13 & 1.29 & 1.79 \\
\hline \multirow{2}{*}{$\begin{array}{l}\text { Surface roughness }(\mathrm{Ra}) \\
\text { after test }[\mu \mathrm{m}]\end{array}$} & wheel & - & - & 0.51 & 0.51 \\
\hline & rail & - & - & 0.75 & 0.69 \\
\hline \multicolumn{2}{|c|}{ Moving Velocity $(\mathrm{mm} / \mathrm{s})$} & \\
\hline \multicolumn{2}{|c|}{ Repeat Count [round trip] } & $1[0.5]$ & $1[0.5]$ & $19[9.5]$ & $10[5]$ \\
\hline
\end{tabular}

Table 2: Test parameters for the rolling measurement

Figure 13 shows the distribution of contact stiffness that was measured with the normal forces of 15 and $25 \mathrm{kN}$. For comparison, the distribution under the static condition (Figure 10) was added to the cross-sectional distribution for both major and minor axes. The peak measured contact stiffness value of the rolling contact condition was higher than that of the static condition. This is because the surface roughness has been decreased by wear or plastic flow by the dynamic contact. The peak stiffness does not occur at the centre, but at the exit side of the contact. This is thought to be 
caused by hysteresis because the exit side corresponds to an unloading process, as has been observed in previous research [25].
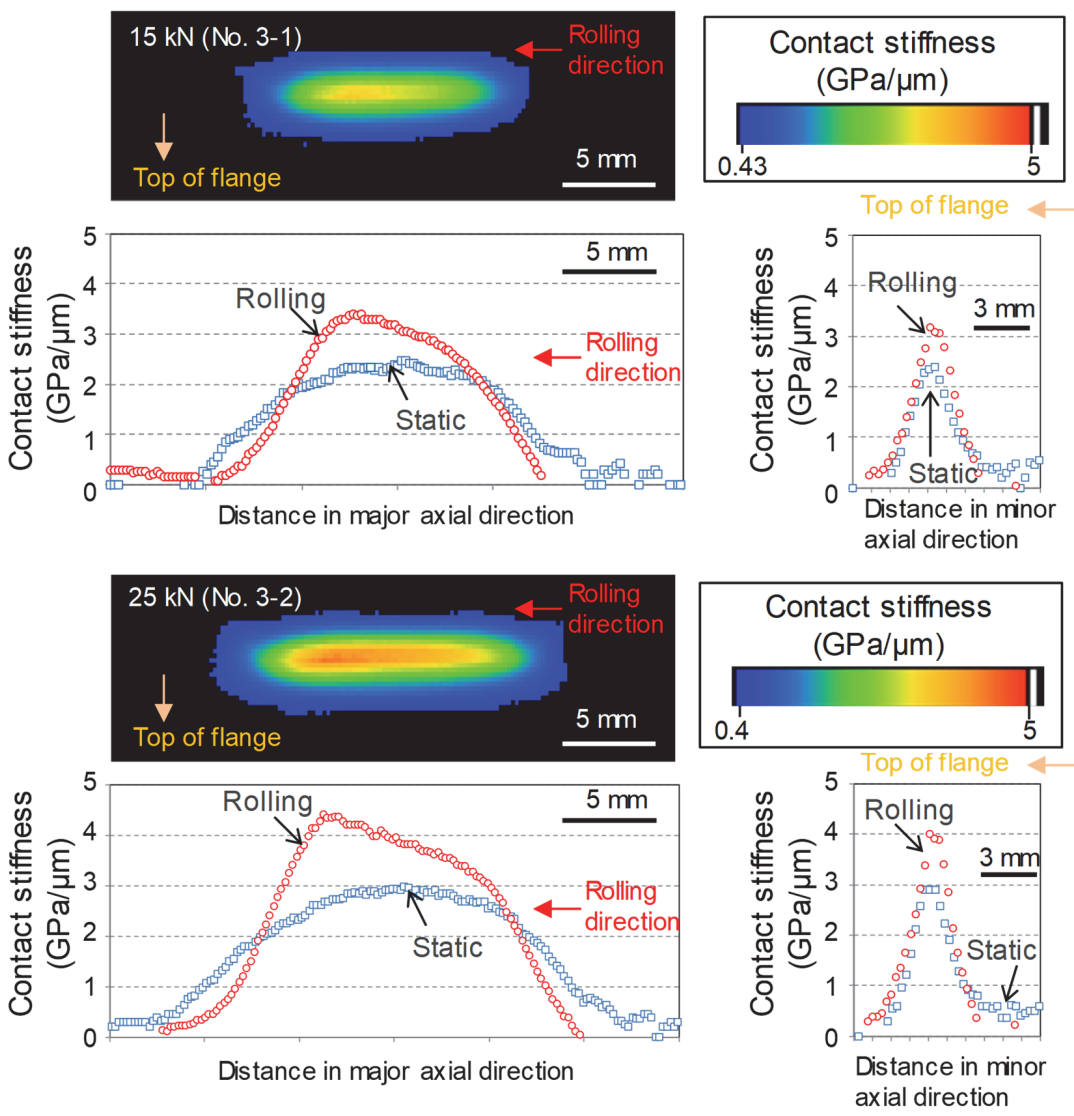

Figure 13: Distribution of contact stiffness that was measured under the rolling contact condition with the normal forces of 15 and $25 \mathrm{kN}$ (No. 3-1: upper, No. 3-2: lower)

The measured length of the major axis for the rolling case was shorter than that for the static case. This is due to the significant difference in the ultrasonic beam paths for the two approaches as shown schematically in Figure 14. For the dynamic case, the ultrasonic wave is focused onto the wheel surface and it remains so throughout the measurement. Any deflection of the wheel surface would cause the incident angle to change and thus introduce some error in the measured reflected wave, reporting a larger drop in $R$ (and hence increase in stiffness) than purely due to interfacial transmission. With the static case, the linear electric stage traverses the array 
following the parallel path of the rail. This measurement therefore is subject to a wider footprint of deflection as both the wheel and rails elastically deform hence the static measurement reports a wider measurement area. It is expected that the wheel was deformed easier than the rail because the back side of the wheel was cut off for the measurement.

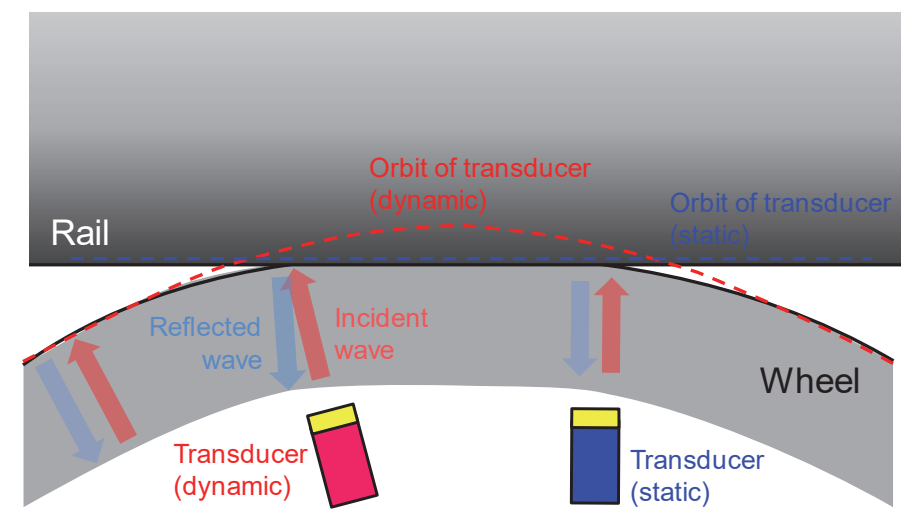

Figure 14: Sketch representing the difference of the static measurement method and dynamic measurement method

Figure 15 shows the representative distribution of contact stiffness that was measured under the cyclic rolling with the normal forces of $15 \mathrm{kN}$ (No. 4-1) and 25 $\mathrm{kN}$ (No. 4-2) after the 1st, 2nd, 9th and 10th contacts. The peak value has increased with each contact. The peak position shift shown in Figure 12 was only seen at the first contact and not seen after the second contact (Figure 14). It is thought that the majority of the plastic flow and reduction in surface roughness was caused during the first rolling process.

Figure 16 shows the relationship between the contact stiffness and the number of contact cycles. Both the mean and sum contact stiffness increased with contact cyclic. The early cycles cause the majority of plastic flow and hence stiffness increase; and it seems to be saturated after around ten contacts. Figure 17 shows the arithmetic average roughness $R a$ (averaged over three measurements) and examples of roughness curve before and after the test. As expected the $R a$ values decrease after each test.

\section{Discussion}

The relationship between the contact stiffness and the lateral force was shown in Section 4.2. This result showed that the contact stiffness increased with increasing lateral force. The comparison between the static and dynamic measurements was shown in Section 4.3. The peak position on the distribution of contact stiffness along with the major axis shifted to the exit side of the contact. Furthermore, the increase in contact stiffness with the cyclic contacts was shown in Section 4.4. These results 
indicate that the contact stiffness increased due to the increase in real contact area caused by the smoothing of the surface by the traction and cyclic contacts.

At the beginning of the test, the asperities on each surface come into contact over a small and therefore high-pressure region. Plastic deformation occurs and the surfaces become more conformal. When the wheel was rolled on the rail a frictional force (tangential force) is created at the interface. This increases the shear stress at the interface and so leads to further plasticity (i.e., the Von Mises or Tresca stress has been increased). This causes a further reduction in roughness. In addition, traction and micro-slip might cause a mild wear process to occur. Both the plastic flow and mild wear would cause an increase in real area of contact and so explains the increased contact stiffness measured.

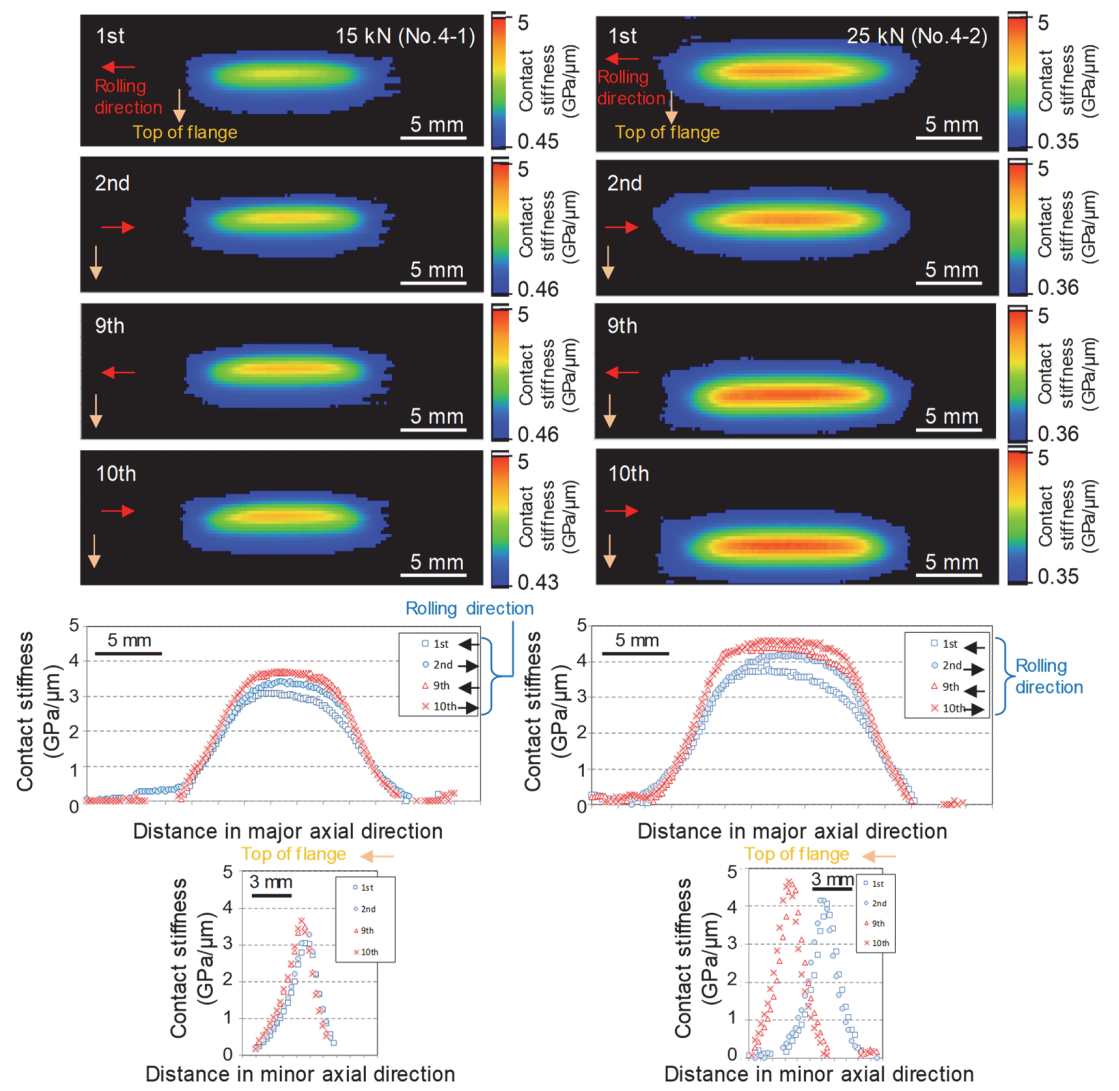

Figure 15: Representative distributions of contact stiffness at the $1^{\text {st }}, 2^{\text {nd }}, 9^{\text {th }}$ and $10^{\text {th }}$ contacts, which were measured under the cyclic rolling contact condition with the normal forces of $15 \mathrm{kN}$ (No. 4-1) and $25 \mathrm{kN}$ (No. 4-2) 


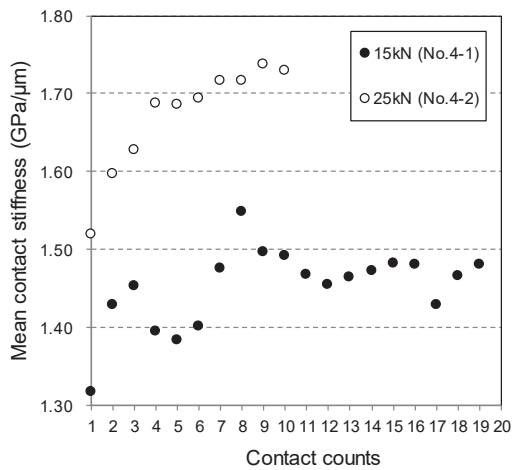

(a) Mean contact stiffness

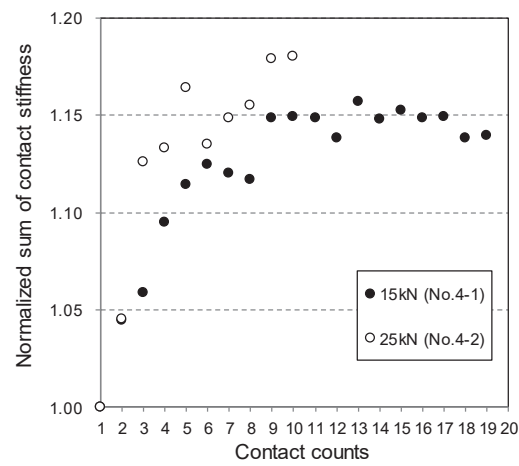

(b) Normalized sum of contact stiffness

Figure 16: The relationship between (a) mean contact stiffness (b) summed contact stiffness, and the number of cyclic contact
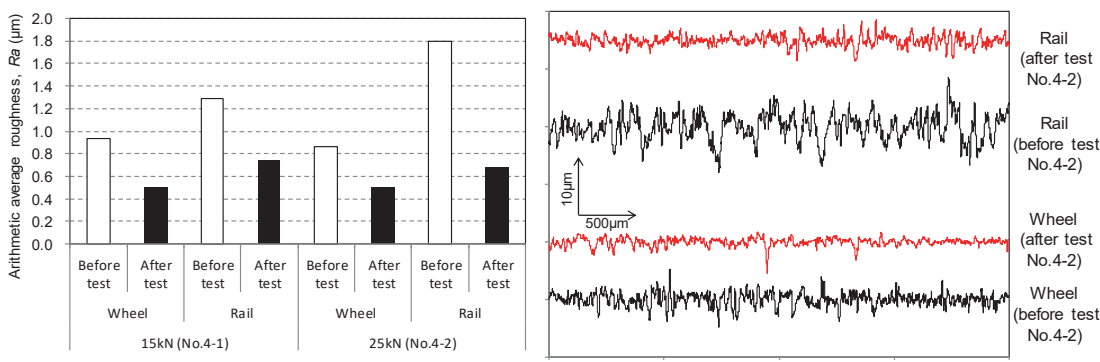

Figure 17: Arithmetic average roughness $R a$ (left hand), and examples of roughness curve before and after the test (right hand).

Generally, the contact area consists of a mixture of elastic and plastic asperity contact. The ratio between the elastically deformed area $A_{e}$ and the plastically deformed area $A_{p}$ depends on the surface topography [26, 27]. This is expressed by the following equation:

$$
N=\int \sigma_{e} a_{e}+\int \sigma_{p} a_{p}
$$

where $\sigma_{p}$ is the contact stress at a plastically deformed asperity, $\sigma_{e}$ is the contact stress at an elastically deformed asperity, $a_{p}$ and $a_{e}$ are the contact area at the plastically and elastically deformed asperity respectively. $A_{p}$ and $A_{e}$ correspond to the sum of $a_{p}$ and the sum of $a_{e}$.

Because $\sigma_{p}$ is larger than $\sigma_{e}$, assuming uniform yield stress among the individual asperities, the real contact area increases with the ratio of $A_{e}$ to $A_{p}, A_{e} / A_{p}$. Since the 
measurements report an increase in contact stiffness, this implies an increase in the value of $A_{e} / A_{p}$, thereby increasing the ratio of $A_{e}$ to $A_{p}$, the sum of $A_{e}$ and $A_{p}$ (Figure 18). Similarly, it has been reported that the value of $A_{e} / A_{p}$ increases with continued sliding [26] and ultrasonic pulses are easier to transmit across smoother interfaces [28].

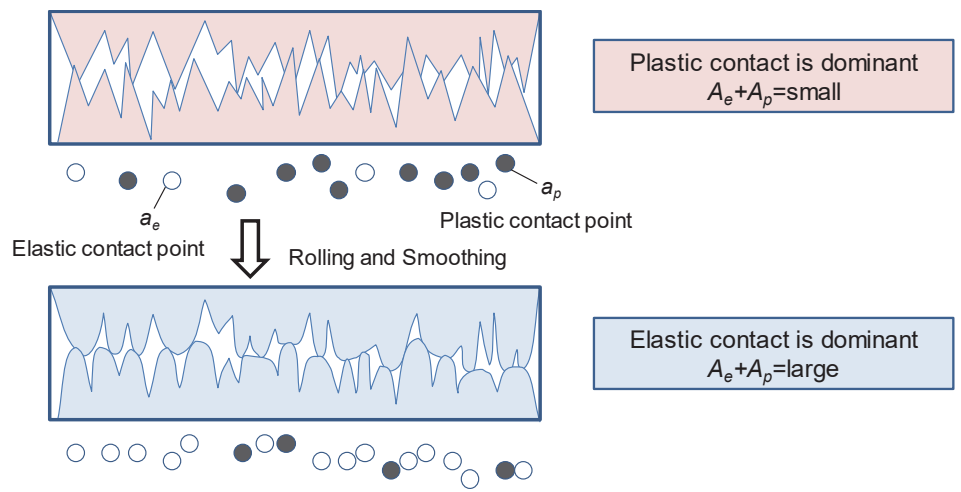

Figure 18: Schematic representation of the increase in the real contact area caused by plastic flow and mild wear.

The real contact area cannot be easily related to the coefficient of friction because any adsorbed layer or a slight amount of moisture has a significant effect. However, adhesion theory suggests that the coefficient of friction is fundamentally dependant on the size of the real contact area, therefore, these results indicate that the tractive force and the cyclic rolling during running-in raise one of the factors responsible for high friction.

\section{Conclusions}

In this study, a measurement technique using reflected ultrasonic waves was applied to evaluate the contact between wheel flange and rail gauge corner. The contact area between the wheel and rail was scanned both statically and dynamically. These tests investigated the influences of tractive force, rolling contact and cyclic contact on the contact condition, particularly contact stiffness. The results and implications of the contact experiments and measurements are as follows:

1. For a given normal load the contact stiffness increased with applied tractive force. This is suggested to be caused by the resulting increased plastic flow, reduction in roughness, and therefore increase in real area of contact.

2. During rolling the peak position of contact stiffness in the contact shifted from the centre to the exit side of the contact. It is thought that this was caused by the change of surface roughness during the rolling.

3. Most plastic flow occurs during the first contact cycle. However, a smaller amount occurs over subsequent cycles saturating after about 10 contacts. 
4. The real contact area cannot be easily related to the coefficient of friction because the state of the near surface layer is highly dependent on the environment. However, it is known that adhesive friction depends strongly on the real area of contact. Therefore, these results indicate that both traction and cyclic rolling during the running-in raise one of the factors associated with elevated wheel-rail friction.

\section{References}

[1] H. Doi, T. Miyamoto, J. Suzumura, J. Nakahashi, H. Chen, T. Ban, "Change in surface condition of turned wheel and effectiveness of lubrication against flange climb derailment”, Quarterly Report of RTRI, 53, 70-76, 2012.

[2] H. Wu, X. Shu, N. Wilson, "Flange climb derailment criteria and wheel/rail profile management and maintenance guidelines for transit operations", TCRP Report 71 Track-Related Research, 5, 2005.

[3] A. Kataori, K. Doi, H. Iijima, S. Momosaki, "The influence of the rail/wheel contact point on friction coefficient", Proceedings of the 16th Jointed Railway Technology Symposium, 2009. (in Japanese)

[4] M. Ishida, T. Nakahara, "Derailment Accident in Hibiya Line and Tribology", Journal of Japanese Society of Tribologists, 46(7), 548-555, 2001. (in Japanese)

[5] B. Koan-Sok, K. Kyogoku, T. Nakahara. "An experimental study of transient traction characteristics between rail and wheel under low slip and low speed conditions", Wear, 265(9), 1417-1424, 2008.

[6] J. Lundmark, E. Kassfeldt, J. Hardell, B. Prakash, "The influence of initial surface topography on tribological performance of the wheel/rail interface during rolling/sliding conditions" Proceedings of the Institution of Mechanical Engineers, Part F: Journal of Rail and Rapid Transit, 223(2), 181-187, 2009.

[7] T. Ban, H. Kakishima, K. Iida, E. Maebashi, H. Ishida, M. Ishida, "A study on the coefficient of friction between rail gauge corner and wheel flange focusing on wheel machining", Proceedings of the $14^{\text {th }}$ International Wheelset Congress, Orlando, U.S.A., October 2004.

[8] J. Lundmark, E. Höglund, B. Prakash, "Running-in behaviour of rail and wheel contacting surfaces", International Conference on Tribology. Vol. 20. 2006.

[9] T. Murakami, "Measurement of contact phenomena", Journal of Japanese Society of Tribologists, 43(7), 572-578, 1998. (in Japanese)

[10] M. Pau, F. Aymerich and F. Ginesu, "Distribution of contact pressure in wheelrail contact area", Wear, 253, 265-274, 2002.

[11] M. Pau, "Estimation of real contact area in a wheel-rail system by means of ultrasonic waves", Tribology International, 36, 687-690, 2003.

[12] M. Pau, "Ultrasonic waves for effective assessment of wheel-rail contact anomalies", Journal of Rail and Rapid Transit, Proceedings of the IMechE Part F, 219, 79-90, 2005.

[13] M. Pau, "Simultaneous subsurface defect detection and contact parameter assessment in a wheel-rail system", Wear, 265, 1837-1847, 2008.

[14] M. Pau, B. Leban, "Ultrasonic assessment of wheel-rail contact evolution exposed to artificially induced wear", Journal of Rail and Rapid Transit, 
Proceedings of the IMechE Part F, 223, 353-364, 2009.

[15] M. B. Marshall, R. Lewis, R. S. Dwyer-Joyce, O. Olofsson, S. Bjorklund, "Measuring wheel/rail contact stresses using ultrasound", Proceedings of the $14^{\text {th }}$ International Wheelset Congress, Orlando, U.S.A., October 2004.

[16] M. B. Marshall, R. Lewis, R. S. Dwyer-Joyce, O. Olofsson, S. Bjorklund, "Experimental characterization of wheel-rail contact patch evolution", ASME Journal of Tribology, 128, 493-503, 2006.

[17] R. S. Dwyer-Joyce, C. Yao and R. Lewis, "Feasibility study for an ultrasonic sensor for monitoring wheel flange contact", Proceedings of 2008 IEEE/ASME Joint Rail Conference JRC2008, Delaware, U.S.A., April 2008.

[18] A. Rovira, A. Roda, M. B. Marshall, H. Brunskill, R. Lewis, "Experimental and numerical modelling of wheel-rail contact and wear", Wear, 271, 911-924, 2011.

[19] R. S. Dwyer-Joyce, C. Yao, R. Lewis, H. Brunskill, "An Ultrasonic sensor for monitoring wheel flange/rail gauge corner contact", Journal of Rail and Rapid Transit, Proceedings of the IMechE Part F, 188-195, 2012.

[20] L. Zhou, H.P. Brunskill, R. Lewis, M.B. Marshall, R.S. Dwyer-Joyce, "Dynamic Characterisation of the Wheel/Rail Contact using Ultrasonic Reflectometry", in J. Pombo, (Editor), "Proceedings of the Second International Conference on Railway Technology: Research, Development and Maintenance", Civil-Comp Press, Stirlingshire, UK, Paper 185, 2014. doi:10.4203/ccp.104.185

[21] S. Fukagai, T. Ban, K. Makino, M. Kuzuta, H. Chen, "The Influence of Repetitive Rolling on the Contact Condition between Wheel and Rail", in J. Pombo, (Editor), "Proceedings of the Third International Conference on Railway Technology: Research, Development and Maintenance", Civil-Comp Press, Stirlingshire, UK, Paper 158, 2016. doi:10.4203/ccp.110.158

[22] M. Schoenberg, 1980. Elastic wave behavior across linear slip interfaces. The Journal of the Acoustical Society of America, 68, 1516-1521.

[23] J. J. Kalker, "Three-Dimensional Elastic Bodies in Rolling Contact", Kluwer Academic Publishers, 1990.

[24] S. Fukagai, T. Ban, K. Makino, M. Kuzuta and H. Chen, "Measurement of contact area between wheel flange and rail gauge corner using ultrasonic technique", 9th International Conference on Contact Mechanics and Wear of Rail/Wheel Systems (CM2012), Chengdu, China, August 27-30, 2012.

[25] R.S. Dwyer-Joyce, B.W. Drinkwater, A.M. Quinn, "The use of ultrasound in the investigation of rough surface interfaces", ASME Journal of Tribology, 123, 8-16, 2001.

[26] J.A. Greenwood and J. B. P. Williamson, "Contact of nominally flat surfaces", Proceedings of the Royal Society of London A: Mathematical, Physical and Engineering Sciences, 295, 300-309, 1966.

[27] W. R. Chang, I. Etsion and D. B. Bogy, "An elastic-plastic model for the contact of rough surfaces”, Journal of Tribology, 109(2), 257-263, 1987.

[28] T. Arakawa, "A study on the transmission and reflection of an ultrasonic beam at machined surfaces pressed against each other", Materials Evaluation, 41, 714$719,1983$. 